\title{
Does ovarian endometrioma affect the number of oocytes retrieved for in vitro fertilization?
}

\author{
Kiran $\mathrm{H}^{1}$, Arikan $\mathrm{DC}^{1}$, Kaplanoglu $\mathrm{M}^{2}$, Bisak $\mathrm{U}^{3}$, Cetin $\mathrm{MT}^{3}$ \\ Kahramanmaras Sutcuimam University, Medical Faculty, Department of Obstetrics and Gynecology, \\ Kahramanmaras, Turkey.drdenizarikan@hotmail.com
}

\begin{abstract}
Objective: To investigate the effects of ovarian endometrioma on the number of oocytes retrieved for in vitro fertilization (IVF).

Background: The presence of endometrioma may be the most important predictor of a poor reproductive outcome. Literature data suggest that ovarian endometriomas might affect the response to ovarian stimulation and oocyte retrieval.

Methods: The present retrospective study evaluates 2,023 women who applied to our center with an infertility complaint. Twenty-nine women with endometriomas (group 1) who were treated with IVF were included in the study. They were compared with 51 women with unexplained infertility (group 2) regarding the number of retrieved oocytes after egg retrieval and number of metaphase II oocytes. The diagnosis of endometrioma was made via ultrasound examination with the identification of low-density cystic masses in the ovaries. The patients underwent a controlled ovarian hyperstimulation $(\mathrm{COH})$ with either the long agonist mini-dose protocol or the multi-dose antagonist protocol.

Results: The incidence of endometrioma in infertile women was found to be $1.4 \%$. The women's ages ranged between 24 and 45 years, and the duration of their infertility ranged between 12 and 216 months. The endometrioma was bilateral in $24 \%$ of the cases. The mean endometrioma diameter was $26.2 \pm 7.3 \mathrm{~mm}$ for the right ovary and $23.2 \pm 6.1 \mathrm{~mm}$ for the left ovary. The average number of retrieved oocytes after egg retrieval in groups 1 and 2 was $12.4 \pm 8.3$ and $12.2 \pm 8.6$, respectively. The average number of metaphase II oocytes in groups 1 and 2 was $8.6 \pm 6.1$ and $9.4 \pm 7.3$, respectively. The number of retrieved oocytes after egg retrieval and the number of metaphase II oocytes in both endometrioma group and unexplained infertile group were similar $(p>0.05)$. Conclusion: Endometrioma did not reduce the number of retrieved oocytes in a $\mathrm{COH}$ cycle for IVF treatment. However it should be noted that the ovarian response is affected by the size of endometriomas, bilaterality, previous surgeries, recurrence, and the patient's age (Tab. 1, Ref. 31). Full Text in PDF www.elis.sk.

Key words: endometrioma, oocyte, infertility.
\end{abstract}

Ovarian endometriomas arise as a result of the development of endometriotic tissues in the ovary. Their inside is generally filled with dense aqua containing hemosiderin, which gives them the color of chocolate. Recently the diagnosis of ovarian endometriomas can be made by transvaginal ultrasonography. The view of widespread low-density echoes within the echogenic cyst wall in the ovary is a typical sonographic feature for the diagnosis of endometrioma (1). The sensitivity of ultrasonography was defined as $80 \%$ in endometrioma diagnosis whereas the specifity was more than $95 \%$ (2).

Endometriomas usually contain thick tar-like fluid; such cysts often densely adhere to the peritoneum of the ovarian fossa and the surrounding fibrosis may involve the tubes and bowel (3). It is

${ }^{1}$ Kahramanmaras Sutcuimam University, Medical Faculty, Department of Obstetrics and Gynecology, Kahramanmaras, Turkey, ${ }^{2}$ Gozde Surgical Medical Center, Department of Obstetrics and Gynecology, Mersin, Turkey, and ${ }^{3}$ Prof Dr M Turan Cetin IVF Center, Department of Obstetrics and Gynecology, Adana, Turkey

Address for correspondence: D.C. Arikan, Kahramanmaras Sütcüimam Üniversitesi, Kadin Hastaliklari ve Dogum Anabilimdali, Yörük Selim Mah. Gazi Mustafa Kuscu Cad. 46050 Kahramanmaras, Turkey.

Phone: +90.505.8022847, Fax: +90.344.2212371 a common pathology among women with endometriosis, and affects $17-44 \%$ of patients with endometriosis (4). It is generally unilateral $(72-81 \%)(5)$. Dede et al evaluated the management of incidental adnexal masses observed at the time of cesarean section. The masses were diagnosed as endometrioma in $4.4 \%$ of cases (6).

Classically, alterations in the production of interleukins, other cytokines, and growth factors are known to occur in the presence of endometriosis in addition to an increase in the number of apoptotic bodies in granulosa cells, changes in the inflammatory response and autoimmune factors and interference with steroid levels, possibly having a negative impact on follicular growth, oocyte quality and implantation rates during assisted reproduction cycles $(7-9)$. The presence of endometrioma may be the most important predictor of a poor reproductive outcome $(10-12)$. Previous reports have suggested that ovarian endometriomas might produce substances that are toxic to oocytes (13) and impair the oocyte development (14).

Literature data have suggested that ovarian endometriomas might affect the response to ovarian stimulation and oocyte retrieval $(14-16)$. In this study, we investigated the number of oocytes retrieved from women who had ovarian endometriomas through in vitro fertilization (IVF) cycles. 


\section{Methods}

The present retrospective study evaluates 2,023 women who applied to our center with infertility complaint. Twenty-nine women who had endometrioma (group 1) and were oriented to IVF treatment were recruited. They were compared with 51 women with unexplained infertility (group 2) regarding the number of retrieved oocytes after egg retrieval and number of metaphase II oocytes. Research ethics approval was obtained from the Ethics Committee of Kahramanmaras Sutcuimam University.

The diagnosis of endometrioma was made via ultrasound examination with the identification of low-density cystic masses in the ovaries. The patients underwent controlled ovarian hyperstimulation $(\mathrm{COH})$ with either long agonist mini-dose protocol or the multi-dose antagonist protocol. Ovarian stimulation was initiated on the 2nd or 3rd days of menstrual cycle with urinary (Menogon, Ferring, Turkey) or recombinant gonadotropins (Puregon, MSD, Turkey or Gonal-F, Serono, Turkey) at the dose of 225 - 300 IU/ day. Follicle development was assessed with serial transvaginal ultrasonography (Siemens-Sonoline Adara, Germany), dose adjustments were made according to ovarian response, and stimulation was carried on through hCG administration. Leuprolide acetate (Lucrin, Abbott, Turkey) $0.5 \mathrm{mg} /$ day was initiated on the $21 \mathrm{st}$ day of previous cycle to women in whom mini-dose long agonist protocol has been performed in order to provide desensitization of pituitary gland. Gonadotropin stimulation was begun on the 2 nd or 3rd day of menstrual cycle and at the same time the agonist dose was reduced in half and carried on through hCG administration. In the antagonist protocol group, gonadotropin stimulation was begun on the 2 nd or 3 rd day of menstrual cycle for $\mathrm{COH}$. Approximately on the 6th day of ovulation induction when the dominant follicle has reached $12-14 \mathrm{~mm}$ in diameter, $0.25 \mathrm{mg} /$ day of ganirelix (Orgalutran, MSD, Turkey) or cetrorelix (Cetrotide, Serono, Turkey) were initiated and carried on through hCG administration. The ovulation trigger was performed with urinary hCG $10.000 \mathrm{IU}$ (Pregnyl, MSD, Turkey) or r-hCG $250 \mathrm{mcg}$ (Ovitrelle, Serono, Turkey) when $\geq 3$ follicles of $\geq 17 \mathrm{~mm}$ were present. Oocyte retrieval was performed under ultrasound guidance $35 \mathrm{~h}$ after hCG injection under general anesthesia.

Poor response was defined as a collection of $<4$ oocytes at oocyte retrieval or cancellation due to an insufficient reaction to the stimulation with gonadotropins ( $<3$ follicles on ultrasound) (17). In all other cases the ovarian response was classified as normal.

Statistical analysis was performed by SPSS 8.0 statistical software, using the Mann Whitney U test. All parameters are given in Table 1 as mean \pm standard deviation, median and minimum-maximum values. $p$ values $<0.05$ were statistically evaluated as significant.

\section{Results}

The incidence of endometrioma in infertile women was found to be $1.4 \%$ ( 29 of 2,023 women). The clinical characteristics of the groups are shown in Table 1. The women's mean age was $32.5 \pm 4.4$ (ranging between 24 and 45 years) in group 1 and 34.1 \pm 4.4 (ranging between 25 and 41 years) in group $2(\mathrm{p}>0.05)$. The mean duration of infertility was $60 \pm 49.8$ months (ranging between 12 and 216 months) in group 1 and 102.1 \pm 52.3 months (ranging between 18 and 192 months) in group 2 ( $p<0.05)$. The mean estradiol, FSH, and endometrial thickness of groups 1 and 2 were similar $(\mathrm{p}>0.05)$.

Endometrioma was bilateral in $24 \%$ of cases. The mean endometrioma diameter was $26.2 \pm 7.3 \mathrm{~mm}$ for the right ovary and $23.2 \pm 6.1 \mathrm{~mm}$ for the left ovary. The average number of retrieved oocytes after egg retrieval in groups 1 and 2 were $12.4 \pm 8.3$ and $12.2 \pm 8.6$, respectively. The average number of metaphase II oocytes in groups 1 and 2 were $8.6 \pm 6.1$ and $9.4 \pm 7.3$, respectively. The numbers of retrieved oocytes and metaphase II oocytes in groups 1 and 2 were similar ( $>0.05$ ). The number of oocytes collected at oocyte retrieval was $\geq 4$ in $93 \%$ of endometrioma group and in $90 \%$ of unexplained infertility group. There was no cancellation due to insufficient reaction to stimulation with gonadotropins for both groups.

\section{Discussion}

One of the factors influencing the success rate during IVF treatment is the ovarian response. In order to compare the ovarian response there are criteria such as the amount of gonadotropin, number of aspirated follicles and number of oocytes, fertilization rate and cycle cancellation $(18,19)$.

The impact of endometriomas on ovarian responsiveness has been studied in some studies. Gupta et al evaluated the ovarian

Tab. 1. The clinical characteristics of groups.

\begin{tabular}{|c|c|c|c|c|c|}
\hline & \multicolumn{2}{|c|}{$\begin{array}{c}\text { Endometrioma Group } \\
(\text { Group 1) }(\mathrm{n}=29)\end{array}$} & \multicolumn{2}{|c|}{$\begin{array}{l}\text { Unexplained İnfertility Group } \\
\text { (Group 2) }(\mathrm{n}=51)\end{array}$} & \multirow[b]{2}{*}{$\mathrm{p}$} \\
\hline & Mean \pm SD & $\begin{array}{c}\text { Median } \\
\text { (Min-Max) }\end{array}$ & Mean $\pm \mathrm{SD}$ & $\begin{array}{c}\text { Median } \\
\text { (Min-Max) }\end{array}$ & \\
\hline Age (years) & $32.5 \pm 4.4$ & $32(24-45)$ & $34.1 \pm 4.4$ & $34(25-41)$ & 0.094 \\
\hline Duration of infertility (months) & $60 \pm 49.8$ & $48(12-216)$ & $102 \pm 52.3$ & $102(18-192)$ & 0.001 \\
\hline $\mathrm{FSH}(\mathrm{mIU} / \mathrm{mL})$ & $8 \pm 4.5$ & $7.6(2.4-25)$ & $8.2 \pm 4.8$ & $6.6(3.2-21.9)$ & 0.708 \\
\hline Endometrial thickness (mm) & $4.5 \pm 3.6$ & $4.5(1-12)$ & $3.7 \pm 2.7$ & $3.5(1-10)$ & 0.437 \\
\hline Total oocyte & $12.4 \pm 8.3$ & $9.00(1-32)$ & $12.2 \pm 8.6$ & $10(0-36)$ & 0.916 \\
\hline MII oocyte & $8.6 \pm 6.1$ & $6(0-25)$ & $9.4 \pm 7.3$ & $7(0-29)$ & 0.764 \\
\hline
\end{tabular}

All parameters are given as mean \pm standard deviation, median and minimum-maximum values in Table 1 . p values $<0.05$ were statistically evaluated as significant. 
reserve and ovarian responsiveness to ovarian stimulation in patients with ovarian endometrioma in a meta-analysis. They found decreased ovarian responsiveness to ovarian stimulation and reduced number of follicles in patients with ovarian endometrioma compared with controls (15). Suzuki et al investigated the effect of endometriosis and the presence of an ovarian endometrioma on outcomes of IVF. Endometriosis affects the oocyte number but not the embryo quality or pregnancy outcome, irrespective of the presence of ovarian endometrioma (14). Somigliana et al reported that the presence of ovarian endometriomas is associated with a reduced responsiveness to gonadotropins. In their study, the numbers of codominant follicles in intact and affected ovaries were 4.0 \pm 2.2 and $3.0 \pm 1.7$, respectively. This difference corresponded to a mean reduction of $25 \%$ (16). Yanushpolsky et al hypothesized that the presence of endometriomas during an IVF cycle adversely affects the oocyte production. Patients with endometriomas produced fewer oocytes than controls. In addition, no significant differences in oocyte parameters were observed in oocytes derived from the endometrioma-containing ovary vs. unaffected contralateral side in the same patient. They concluded that the number of oocytes retrieved may be affected adversely in the presence of endometriomas (20). Dos Reis et al reported that the presence of endometrioma at the time of aspiration caused a decline in ovarian reserves as demonstrated by the smaller number of retrieved follicles and aspirated oocytes when compared to the contralateral healthy ovary (21). On the other hand, Olivennes et al did not observe any adverse effects of endometriomas on oocyte quality in the retrospective review of their IVF data. Nevertheless, they consistently observed a decreased number of oocytes retrieved when severe mechanical infertility was associated with endometriosis (22). Recently, it has been shown that the presence of ovarian endometrioma in a $\mathrm{COH}$ cycle for IVF treatment is not associated with a reduced number of oocytes retrieved from the affected ovary (23).

Various treatment modalities have been used to achieve conception in infertile women having endometrioma, including expectant management, cystectomy, medical therapy, $\mathrm{COH}$, and assisted reproductive technologies (ART). Kennedy et al recommend that histology should be obtained to exclude malignancy in cases of endometrioma of more than $3 \mathrm{~cm}$ in diameter (3), and cystectomy is generally used for endometriomas of more than $3 \mathrm{~cm}$ in diameter before ART (24).

The impact of surgical treatment of endometriomas on IVF results and ovarian response are controversial. Dlugi et al suggested that the presence of an ovarian endometrioma had an adverse effect on IVF outcome and suggested that patients with ovarian endometriomas should have them removed prior to undergoing IVF (25). On the other hand, Ho et al reported that surgery for ovarian endometrioma may damage the ovarian reserve. It potentially results in poor ovarian response to $\mathrm{COH}$ compared to the response of the contralateral normal ovary in the same individual (26). Demirol et al investigated the effect of conservative surgery of ovarian endometriomas before an ICSI cycle. The patients were prospectively randomized into two groups; group 1 underwent a conservative ovarian surgery before the ICSI cycle and group 2 underwent the ICSI cycle directly. They reported that ovarian sur- gery resulted in longer stimulation, higher FSH requirement, and lower oocyte number (27).

Suganuma et al analyzed the IVF outcomes with or without pretreatment in patients with endometrioma in order to determine what pretreatment should be performed for ovarian endometrial cyst before IVF. Infertile women with endometrioma who underwent IVF were divided into three groups, including patients who had received laparotomy or laparoscopy, those for whom the endometrioma content had been aspirated and treated with or without alcohol fixation, and patients who did not undergo pretreatment. The results showed that the pretreatment for endometrioma reduced the number of retrieved oocytes (28). Pabuccu et al detected that all patients with endometriomas had significantly lower numbers of MII oocytes compared with those in patients with tubal factor infertility. They proposed that aspiration of endometriomas before $\mathrm{COH}$ neither reduced the amount of gonadotropins nor increased the number of follicles of $>17 \mathrm{~mm}$, as well as the number of MII oocytes (29). Almog et al compared the response of operated and non-operated ovaries to gonadotropin stimulation in 38 women who had had excision of ovarian endometrioma. The antral follicle count, numbers of dominant follicles, and number of oocytes collected in the operated ovaries were significantly lower than those in the non-operated ovaries, suggesting a reduced ovarian reserve after excision of ovarian endometrioma (30). Nonsurgical treatment might be a better option to avoid the reducing of the ovarian response in women with endometrioma (31).

In our study, the average number of retrieved oocytes after egg retrieval was $12.4 \pm 8.3$ and the average number of metaphase II oocytes was $8.6 \pm 6.1$ in women with ovarian endometrioma. On the basis of these findings, we may conclude that the presence of endometriomas during $\mathrm{COH}$ for assisted reproduction does not reduce the number of collected oocytes. Poor response was defined as a collection of $<4$ oocytes at oocyte retrieval or cancellation due to an insufficient reaction to stimulation with gonadotropins (17). Most women with endometriomas who were medically treated prior to IVF responded well to ovarian stimulation in our study.

\section{Conclusion}

Endometrioma did not reduce the number of retrieved oocytes in a $\mathrm{COH}$ cycle for IVF treatment.

We suggest that when women suffer from endometrioma it might be a better option to encourage the couples to apply directly for ART as the first line of treatment for achieving a higher ovarian response and avoiding the ovarian damage and other complications of surgery. However, it should be noted that the ovarian response is affected by the size of endometriomas, bilaterality, previous surgeries, recurrence, and the patient's age.

\section{References}

1. Patel MD, Feldstein VA, Chen DC, Lipson SD, Filly RA. Endometriomas: diagnostic performance of US. Radiology 1999; 210 (3): 739-745.

2. Spaczynski RZ, Duleba AJ. Diagnosis of endometriosis. Semin Reprod Med 2003; 21 (2): 193-208. 
3. Kennedy S, Bergqvist A, Chapron C et al. on behalf of the ESHRE Special Interest Group for Endometriosis and Endometrium Guideline Development Group.ESHRE guideline for the diagnosis and treatment of endometriosis. Hum Reprod 2005; 20 (10): 2698-2704.

4. Redwine DB. Ovarian endometriosis: a marker for more extensive pelvic and intestinal disease. Fertil Steril 1999; 72 (2): 310-315.

5. Al-Fozan H, Tulandi T. Left lateral predisposition of endometriosis and endometrioma. Obstet Gynecol 2003; 101: 164-166.

6. Dede M, Yenen MC, Yilmaz A, Goktolga U, Baser I. Treatment of incidental adnexal masses at cesarean section: a retrospective study. Int J Gynecol Cancer 2007; 17 (2): 339-341.

7. Ayers JWT, Birenbaum DL, Jiaram Menon KM. Luteal phase dysfunction in endometriosis: Elevated progesterone levels in peripheral and ovarian veins during the follicular phase. Fertil Steril 1987; 47: 925-929.

8. Pellicer A, Oliveira N, Ruiz A, Remohi J, Simon C. Exploring the mechanism(s) of endometriosis-related infertility: An analysis of embryo development and implantation in assisted reproduction. Hum Reprod 1995; 10: $91-07$.

9. Lucena E, Cubillos J. Immune abnormalities in endometriosis compromising fertility in IVF-ET patients. J Reprod Med 1999; 44: 458-464.

10. Matson PL, Yovich JL. The treatment of infertility associated with endometriosis by in vitro fertilization. Fertil Steril 1986: 46: 432-434.

11. Yovich JL, Matson PL, Richardson PA, Milliard C. Hormonal profiles and embryo quality in women with severe endometriosis treated by in vitro fertilization and embryo transfer. Fertil Steril 1988: 50: 308-313.

12. Dlugi AM, Loy RA, Dieterle S, Bayer SR, Seibel MM. The effect of endometriomas on in vitro fertilization outcome. J In Vitro Pert Embryo Transfer 1989: 6: 338-341.

13. Yanushpolsky EH, Best CL, Jackson KV, Clarke RN, Barbieri RL, Hornstein MD. Effects of endometriomas on oocyte quality, embryo quality and pregnancy rates in in vitro fertilization cycles: a prospective, casecontrolled study. J Assist Reprod Genet 1998; 15: 193-197.

14. Suzuki T, Izumi S, Matsubayashi H, Awaji H, Yoshikata K, Makino T. Impact of ovarian endometrioma on oocytes and pregnancy outcome in in vitro fertilization. Fertil Steril 2005; 83 (4): 908-913.

15. Gupta S, Agarwal A, Agarwal R, Loret de Mola JR. Impact of ovarian endometrioma on assisted reproduction outcomes. Reprod Biomed Online 2006; 13 (3): 349-360.

16. Somigliana E, Infantino M, Benedetti F, Arnoldi M, Calanna G, Ragni G. The presence of ovarian endometriomas is associated with a reduced responsiveness to gonadotropins. Fertil Steril 2006; 86 (1): 192-196.

17. Bancsi LF, Broekmans FJ, Eijkemans MJ, de Jong FH, Habbema JD, te Velde ER. Predictors of poor ovarian response in in vitro fertilization: a prospective study comparing basal markers of ovarian reserve. Fertil Steril 2002; 77 (2): 328-336.

18. Mukherjee T, Copperman AB, Lapinski R, Sandler B, Bustillo M, Grunfeld L. An elevated day three follicle-stimulating hormone: luteinizing hormone ratio (FSH: LH) in the presence of a normal day 3 FSH predicts a poor response to controlled ovarian hyperstimulation. Fertil Steril 1996; 65: 588-592.
19. Speroff L, Glass RH, Kase NG, ed. Clinical Gynecologic Endocrinology and Infertility. 6th Edition. Lippincott Williams \&Wilkins, Baltimore, USA, 1999, 1133-1148.

20. Yanushpolsky EH, Best CL, Jackson KV, Clarke RN, Barbieri RL, Hornstein MD. Effects of endometriomas on ooccyte quality, embryo quality, and pregnancy rates in in vitro fertilization cycles: a prospective, case-controlled study. J Assist Reprod Genet 1998; 15 (4): 193-197.

21. dos Reis RM, Corrêa ILA, De Ângelo AG, Manetta LA, de Moura MD, Ferriani RA. Short Communication: In Vitro Fertilization in Patients with Ovarian Endometrioma. Journal of Ass Reprod Genetics 2004; 21 (8): 311-314.

22. Olivennes F, Feldberg D, Liu HC, Cohen J, Moy F, Rosenwaks Z. Endometriosis: A stage by stage analysis-The role of in vitro fertilization. Fertil Steril 1995; 64 (2): 392-398.

23. Almog B, Shehata F, Sheizaf B, Tan SL, Tulandi T. Effects of ovarian endometrioma on the number of oocytes retrieved for in vitro fertilization. Fertil Steril 2010 Apr 6. [Epub ahead of print].

24. Beretta P, Franchi M, Ghezzi F, Busacca M, Zupi E, Bolis P. Randomized clinical trial of two laparoscopic treatments of endometriomas: cystectomy versus drainage and coagulation. Fertil Steril 1998; 70 (6): $1176-1180$.

25. Dlugi AM, Loy RA, Dieterle S, Bayer SR, Seibel MM. The effect of endometriomas on in vitro fertilization outcome. J In Vitro Fert Embryo Transf 1989; 6 (6): 338-341.

26. Ho HY, Lee RK, Hwu YM, Lin MH, Su JT, Tsai YC. Poor response of ovaries with endometrioma previously treated with cystectomy to controlled ovarian hyperstimulation. J Assist Reprod Genet 2002; 19(11): 507-511.

27. Demirol A, Guven S, Baykal C, Gurgan T. Effect of endometrioma cystectomy on IVF outcome: a prospective randomized study. Reprod Biomed Online 2006; 12 (5): 639-643.

28. Suganuma N, Wakahara Y, Ishida D, et al. Pretreatment for ovarian endometrial cyst before in vitro fertilization. Gynecol Obstet Invest 2002; 54 ( Suppl 1): 36-40.

29. Pabuccu R, Onalan G, Goktolga U, Kucuk T, Orhon E, Ceyhan T. Aspiration of ovarian endometriomas before intracytoplasmic sperm injection. Fertil Steril 2004; 82 (3): 705-711.

30. Almog B, Sheizaf B, Shalom-Paz E, Shehata F, Al-Talib A, Tulandi T. Effects of excision of ovarian endometrioma on the antral follicle count and collected oocytes for in vitro fertilization. Fertil Steril 2010 Feb 26. [Epub ahead of print].

31. Geber S, Ferreira DP, Spyer Prates LF, Sales L, Sampaio M. Effects of previous ovarian surgery for endometriosis on the outcome of assisted reproduction treatment. Reprod Biomed Online 2002; 5: 162-166.

Received November 2, 2010. Accepted May 5, 2012. 